Article

\title{
Optimization of Phasor Measurement Unit (PMU) Placement in Supervisory Control and Data Acquisition (SCADA)-Based Power System for Better State-Estimation Performance
}

\author{
Mohammad Shoaib Shahriar * (1), Ibrahim Omar Habiballah and Huthaifa Hussein \\ Department of Electrical Engineering, King Fahd University of Petroleum \& Minerals (KFUPM), Dhahran 31261, \\ Saudi Arabia; ibrahimh@kfupm.edu.sa (I.O.H.); g201410860@kfupm.edu.sa (H.H.) \\ * Correspondence: shoebeee05@gmail.com; Tel.: +966-503552319
}

Received: 9 February 2018; Accepted: 1 March 2018; Published: 6 March 2018

\begin{abstract}
Present-day power systems are mostly equipped with conventional meters and intended for the installation of highly accurate phasor measurement units (PMUs) to ensure better protection, monitoring and control of the network. PMU is a deliberate choice due to its unique capacity in providing accurate phasor readings of bus voltages and currents. However, due to the high expense and a requirement for communication facilities, the installation of a limited number of PMUs in a network is common practice. This paper presents an optimal approach to selecting the locations of PMUs to be installed with the objective of ensuring maximum accuracy of the state estimation (SE). The optimization technique ensures that the critical locations of the system will be covered by PMU meters which lower the negative impact of bad data on state-estimation performance. One of the well-known intelligent optimization techniques, the genetic algorithm (GA), is used to search for the optimal set of PMUs. The proposed technique is compared with a heuristic approach of PMU placement. The weighted least square (WLS), with a modified Jacobian to deal with the phasor quantities, is used to compute the estimation accuracy. IEEE 30-bus and 118-bus systems are used to demonstrate the suggested technique.
\end{abstract}

Keywords: phasor-measurement unit; optimal PMU placement; state estimation; weighted least square; critical measurement; genetic algorithm; heuristic approach

\section{Introduction}

The classical state estimation of a power system is based upon measurements collected from a supervisory control and data acquisition (SCADA) system. Such a system cannot capture measurements of fast and dynamic phenomena. Another drawback of SCADA measurements is that they do not include the phase angle of bus voltages or line currents. Alternatively, real-time voltage as well as current phasors are supplied by phasor measurement units (PMUs). These are digital devices with very high sampling rate and precision, synchronized by receiving a common timing signal from a global positioning satellite (GPS) clock [1]. Compared with traditional SCADA data, PMU measurements achieve a synchronization precision of one microsecond and magnitude accuracy of $0.1 \%$ [2].

Over time, greater numbers of PMUs have become necessary to improve monitoring, control and the protection of the power system. Due to the high price of PMUs and the cost of communication services connected with them, it is not possible to place a unit at each bus of the network. Rather, a certain number of PMUs are required to be placed at certain locations which make the system fully observable. This is known as the optimal placement of PMUs problem and there are 
numerous publications in this field. References [3-7] give an extensive review of the problem in the literature. The review papers present all the relevant work in the field of PMU optimization along with further information on objective functions, constraints, and optimization tools tried by researchers. Most of these papers, however, exclude conventional measurements from the formulation and only PMU measurements are considered for finding the optimal locations and the smallest number of PMUs that render the power system observable and, therefore, can be used for state estimation.

State estimation using only PMU measurements is not practical for most of the power systems in the current period due to cost considerations and needs [6,8]. A more realistic approach is to use conventional SCADA measurements with PMU measurements to enhance the performance and accuracy of state estimation. Some of this work is presented in [9-12] introducing techniques for combining these measurements with supporting theory and simulations. In [13], an incremental PMU placement approach is applied to reduce the uncertainties of state estimation based on conventional SCADA measurements. This incremental algorithm is adopted from [14] where the covariance of the state-error vector-or the diagonal elements of the gain matrix inverse in the WLS method-is used to assess the quality of the estimation. The voltage phasor of PMU is considered in [13] and the current phasor is excluded. The authors of [15] established the PMU placement problem as an optimal experiment design which has a class of renowned optimality criteria in statistics. Reference [16] presents a comparative study between the approaches of PMU inclusion in the estimation process. An approach to finding PMU locations for the least absolute value (LAV) algorithm is presented in [17] which can detect bad data without finding out the critical sets. In this paper, the concept of "branch PMU" is also introduced which provides a single voltage-phasor and a single current-phasor measurement. Paper [18] proposes an optimal strategy of PMU placement for maximizing the average detection performance when multiple line outages occur. The algorithm proposed in [19] can make the system observable, detect bad-data and is capable of working with mixed measurement cases. The optimization technique of [20] deals with the gain matrix and finds the rank of the Jacobian, thereby reaching the optimal solution in only 3-4 iterations by shortening the constraint equations. Here, algorithms for observability and bad-data detection are presented separately.

Paper [21] proposes a heuristic PMU placement technique to enhance the state-estimation precision which is assessed by the performance indicator of average mean average percentage error (MAPEave). But the approach has the limitation of falling in a local minimum solution of a PMU set as it selects PMU locations one by one. None of the intelligent techniques has been used yet to search for the limited numbers of optimal PMU locations to ensure the maximum estimation performance.

This paper presents an optimization strategy of selecting any limited number of PMU locations in the power system. The objective of the method is to improve the estimation accuracy as well as to cover the critical regions of the system with accurate PMU meters. The use of the well-known evolutionary programming technique, binary genetic algorithm (BGA), for this purpose makes the idea efficient. In addition, this paper compares the proposed strategy with the heuristic approach presented in reference [21] to prove its superiority. A modified WLS estimator which is capable of dealing with mixed measurements (SCADA + PMU) is used in this work for calculating the error indicator of estimation, which is also the fitness value of the BGA. IEEE 30-bus and 118-bus systems are used to present the proposed method of PMU placement.

If any power system which is installed with SCADA meters plans to install a restricted number of PMUs to improve the state estimation performance, the proposed method of PMU deployment would be an ideal option to follow. The proposed technique of PMU placement also takes care of the issue of critical measurements. The presence of critical measurements hampers the estimation performance badly if the bad data come from critical measurements or are associated with them. Covering the critical zones during PMU placement will surely ensure the robustness of the estimation performance of the power system. To the best of the authors' knowledge, this is a novel approach to a PMU placement strategy, even though a significant amount of work has been done in the field of PMU placement previously. 
To outline this paper, Section 2 explains the modified WLS algorithm which can estimate the power-system states with the presence of both SCADA and PMU meter readings. Section 3 presents the heuristic approach of PMU placement, which has been adopted for comparison purposes. Section 4 displays the problem formulation of the optimal PMU placement as well as the BGA approach of solving that problem. Section 5 demonstrates the preparation of different test cases in detail. Elaborate simulation results are presented in Section 6 along with the significance of covering the critical zones in the optimization problem. Section 7 concludes the paper.

\section{Modified Weighted Least Square with Phasor Measurements}

The weighted least square is an estimation problem that searches for a solution from the over-defined matrix equations when there are few states and many measurements in the power system [22,23]. A conventional state estimator employs SCADA measurements whose relationship with system states are as follows:

$$
z_{i}=h_{i}(x)+e_{i}
$$

$z_{i}$ : is SCADA measurement vector.

$h_{i}(x)$ : corresponds to a non-linear function vector which relates measurements to states.

$e_{i}$ : is the error vector between measured and estimated value.

Power system state estimation is a system of overdetermined non-linear equations and should be solved as an unconstrained problem. The weighted least square has the objective function which minimizes the sum of the square of the residuals:

$$
\min f(x)=\sum_{i=1}^{N} W_{i}^{2}\left|h_{i}(x)-z_{i}\right|^{2}
$$

To minimize the function, an iterative procedure followed by Newton's method is used. States will be updated in each iteration until it satisfies the stopping criteria:

$$
x_{k+1}=x_{k}+\Delta x=x_{k}+\left[[H]^{T}[R]^{-1}[H]\right]^{-1}[H]^{T}[R]^{-1}\left[\begin{array}{c}
z_{1}-h_{1}(x) \\
z_{2}-h 2(x) \\
\vdots \\
z_{m}-h_{m}(x)
\end{array}\right]
$$

By considering a partial derivative of $h(x)$ with respect to state vector $x$, a Jacobian matrix [H] will be acquired. $\Delta x$ is the measurement mismatch and is used as a counter step for the next iteration. $[R]$ is the error covariance matrix of SCADA measurements. The iterative procedure stops when $\Delta x$ goes below a specific low threshold number.

When a PMU is installed, phasor measurements are present in the measurement series and, hence, the estimator should be modified and updated accordingly. The Jacobian matrix should include the elements containing the derivatives of voltage and current phasors with respect to the system states. The formulation of WLS is modified in this paper to run the estimation properly with a mixed measurement (SCADA and PMU) system. The modified Jacobian used for the paper is as follows: 


$$
H=\left[\begin{array}{cc}
\frac{\delta P_{\text {inj }}}{\delta \theta} & \frac{\delta P_{\text {inj }}}{\delta V} \\
\frac{\delta P_{\text {flow }}}{\delta \theta} & \frac{\delta P_{\text {flow }}}{\delta V} \\
\frac{\delta Q_{\text {inj }}}{\delta \theta} & \frac{\delta Q_{\text {inj }}}{\delta V} \\
\frac{\delta Q_{\text {flow }}}{\delta \theta} & \frac{\delta Q_{\text {flow }}}{\delta V} \\
\frac{\delta V}{\delta \theta} & \frac{\delta V}{\delta V} \\
\frac{\partial I_{F, \text { Real }[i j]}}{\partial \theta} & \frac{\partial I_{F, \text { Real }[i]]}}{\partial V m} \\
\frac{\left.\partial I_{F, I m a g}[i]\right]}{\partial \theta} & \frac{\partial I_{F, I m a g}[i j]}{\partial V m}
\end{array}\right]
$$

The $\mathrm{H}$ matrix of Equation (4) includes the partial derivatives of power-injections $\left(P_{i n j}, Q_{i n j}\right)$, power flows $\left(P_{\text {flow }}, Q_{\text {flow }}\right)$, voltage magnitudes $(V)$ and current phasors $\left(I_{F, \text { Real }}, I_{F, \text { imag }}\right)$ with respect to two state variables: voltage magnitude $(V)$ and phase angle $(\theta)$.

The PMUs are incorporated in the state-estimation algorithm with the following assumptions [11]:

- The phasor measurements and the traditional measurements are taken at the same snapshot-there is no time skew between them.

- A PMU should be always available at the slack bus so that the reference angle of both measurements is the same.

- When a PMU is installed at a certain bus, it can read the bus voltage phasor in addition to all the branch current phasors connected to that bus and flowing away from that bus.

MATPOWER [24], which is a package of MATLAB (R2017b, MathWorks, Natick, MA, USA) files for solving power-system operations such as state estimation and power flow, is utilized in our work since it is designed as a simulation tool for educators and researchers that is simple to use and modify.

\section{Heuristic Approach to Optimization}

For this approach, we will adopt the algorithm presented in reference [21] since this considers enhancing the estimation accuracy as the principal purpose of deploying PMUs in a power system.

This assumes $p$ PMUs have to be placed in a power system of $\mathrm{n}$ buses $(p<n)$. After each installation of a PMU, a state-estimation algorithm is executed, and the accuracy of the estimation is calculated by the following normalized cumulative error (NCE) indicator:

$$
\begin{gathered}
N C E=\frac{1}{2 \times n}\left(\sum_{i=1}^{2 n} \mid \text { Actual }_{i}-\text { Estimated }_{i} \mid\right) \\
N C E=\frac{1}{2 \times n}\left(\sum_{i=1}^{n}\left[\mid \text { Actual }_{V m_{i}}-\text { Estimated }_{V m_{i}}|+| \text { Actual }_{V a_{i}}-\text { Estimated }_{V a_{i}} \mid\right]\right)
\end{gathered}
$$

where Actual $_{i}$ represents all the load-flow actual values, and Estimated ${ }_{i}$ are the corresponding estimated values provided by the estimator. Minimum NCE values will ensure the maximum efficiency of state estimation. The performance indicator NCE is the absolute summation of all the differences between the actual values and estimated values of the power-system states (voltage magnitudes $(\mathrm{Vm})$ and phase angles (Va)) as shown in Equations (5) and (6).

This algorithm assumes that the system is already observable by traditional measurements and PMUs are to be placed to improve the estimation accuracy. The slack bus, usually bus 1 , in the network is the reference bus where a PMU is installed to provide an accurate reference for other buses. Before describing the algorithm procedure, the variables used are defined as follows [21]:

- $\quad z$ : the measurement vector with white noise;

- $\quad R$ : the vector of measurement error variance; 
- $\quad p$ : number of PMUs required to be placed in the system;

- AllBus: contains all the numbers of candidate buses;

- BusWithPMU: includes the bus numbers which have been assigned a PMU;

- BusToPlace: the vector which includes the bus numbers that will be equipped with PMUs. It is acquired by eliminating BusWithPMU from AllBus;

- Num: the length of BusToPlace vector;

- BusNum: the parameter that includes the bus number that will be equipped with a PMU;

- KnownPMUBus: composed by merging BusWithPMU vector and BusNum variable;

- Ind_array: contains NCE values of state estimation.

This heuristic algorithm places PMUs incrementally bus by bus. For each placement, it tests all the candidate buses comprehensively and the bus with lowest indicator value is chosen. The algorithm is explained in reference [21] in detail with a different performance indicator of SE. The flowchart of this algorithm is displayed in Figure 1 below.

This algorithm solves the placement problem by providing both the number and bus location of PMUs needed to reach a desired estimation accuracy. It is suitable for any system configuration without the need to adjust the code. It can also be applied to a certain area of the system rather than the whole system. This can be simply achieved by selecting the required buses in vector AllBus.

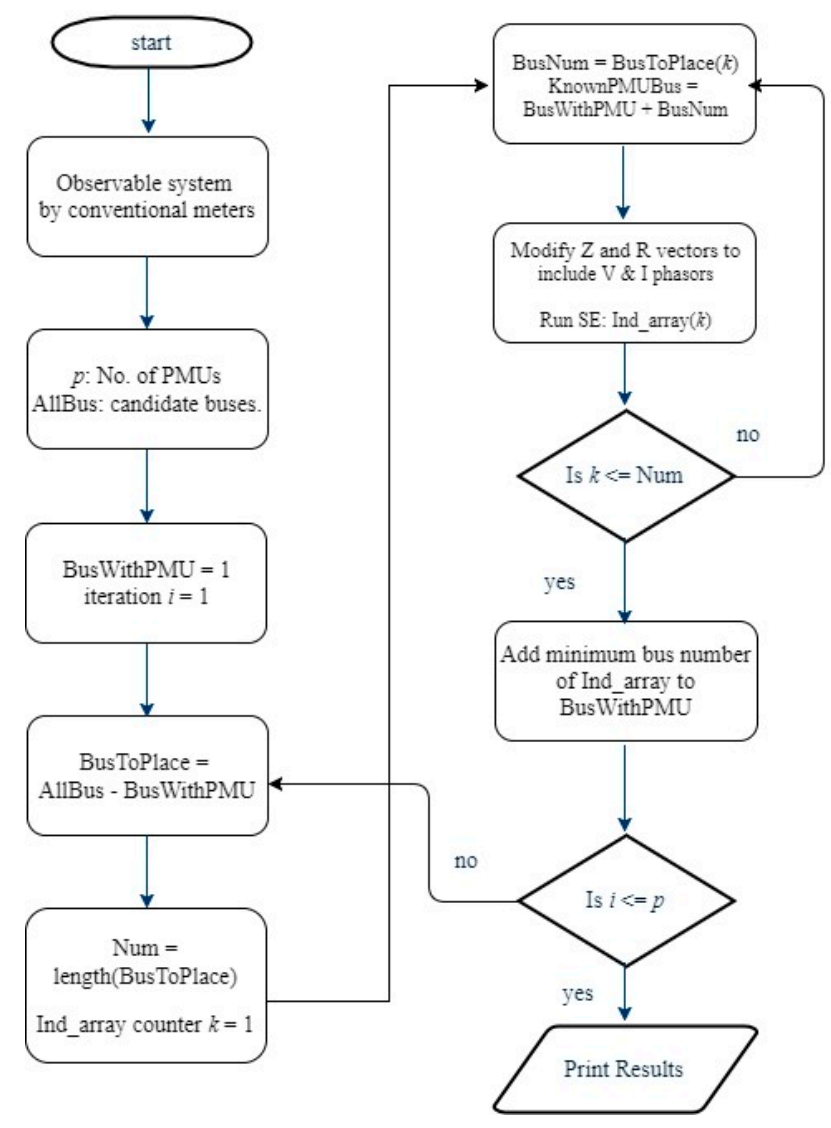

Figure 1. Flowchart of heuristic optimal PMU placement (OPP) algorithm.

\section{Optimization with Genetic Algorithm}

\subsection{Problem Formulation of Optimal Phasor Measurement Unit (PMU) Placement:}

The objective of the optimization problem is to get a set of PMU locations that will ensure the best estimation performance. The number of PMU locations to optimize could be any number within the 
range of available bus locations. This is not a problem like minimizing or maximizing an objective function but of finding out the appropriate locations for the intended number of PMUs to be placed in a power system that ensures the minimum estimation error.

So, the problem formulation could be stated as follows:

Find $X$

$$
\left(x_{1}, x_{2}, \cdots, x_{n}\right) \in X
$$

where $x$ denotes the bus locations to be installed with PMUs and $n$ represents the maximum number of PMUs to install.

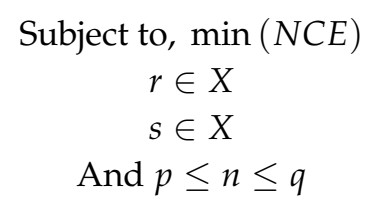

$r$ : is the reference bus which must be present in the optimal set X. s: represents the buses that cover the critical measurements for a given measurement set. For example, if it is found that the SCADA-meter measurement set has three critical measurements such as $P F \_i-j, Q F \_i-j$ and $P G i$, then bus $i$ will be considered as the member of $s$ which must be chosen in the optimal solution set, X. $p$ and $q$ are the minimum and maximum bus locations to be considered in optimization problem. For a 30-bus power system, the values for $p$ and $q$ will be 1 and 30, respectively.

The concept of normalized residuals (NR) is used to identify the critical locations. Zero NR for a measurement denotes a critical measurement $[25,26]$.

So, the proposed approach of PMU placement calculates the residuals for each available measurement, identifies the critical ones, selects the elitism strategy to cover the critical measurements along with the slack bus and, finally, goes for the main optimization process by the genetic algorithm. Figure 2 shows how the elitism strategy is set before going to the main process of optimization by BGA.

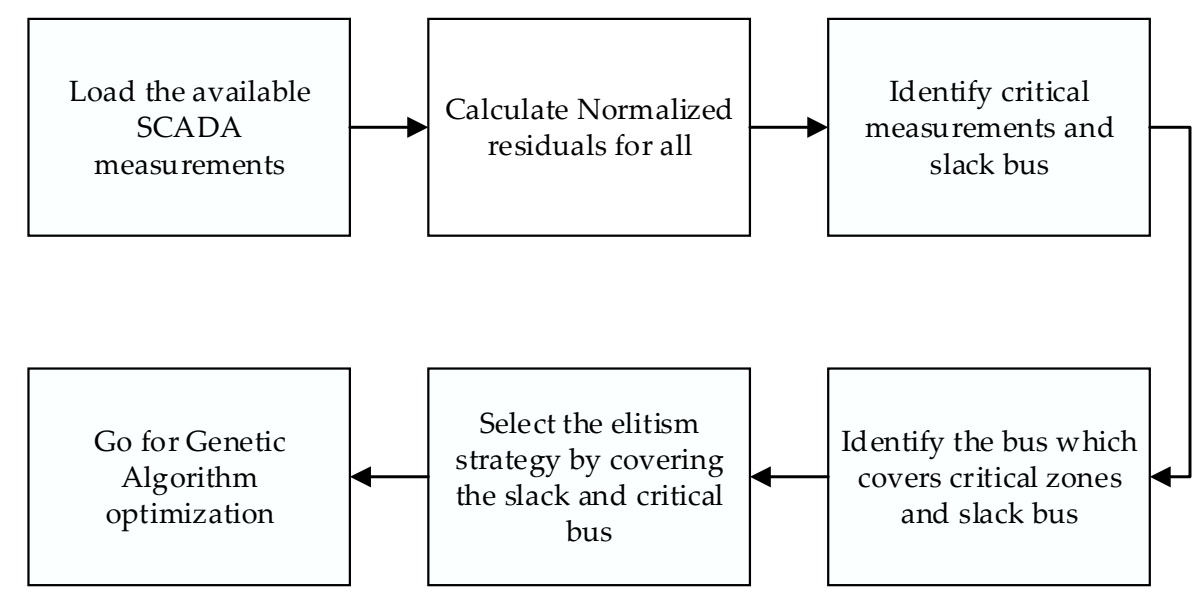

Figure 2. Flow diagram of optimization process.

\subsection{Basic Working Principle of the Binary Genetic Algorithm (BGA)}

The binary version of the genetic algorithm (GA) is an intelligent exploitation search algorithm based on the evolutionary concepts of natural selection and genetics. GA, as a part of evolutionary computing, mimics the idea of Darwin's "survival of the fittest" in reaching a global solution for an optimization problem. In the working procedure of BGA, a set of population is selected randomly. Different genetic operators act on the population to search for the global solution set. Genetic diversity is maintained by the operators throughout the entire process as it is needed for the natural evolution. 
Three main operators of BGA are as follows $[27,28]$ :

1. Selection or reproduction: this phase generates the chromosomes or parents based on their fitness value. Error indicator of the state estimation is used as the fitness for this work. A lower indicator represents better chromosome or a PMU location set.

2. Crossover: this creates the children or off-springs from the parents with the view that the child could be fitter than the parents. Off-springs are formed by taking the best characteristics from both parents based on a user-defined probability. Crossover could be done on a single or multiple point based on the size of the chromosomes.

3. Mutation: this works on the off-springs, checking each bit individually to search for a better solution.

Some issues such as selection of initial population size, different probability values, selection of elite chromosome, maximum number of iterations, stopping criteria etc. play important roles in BGA [22].

Figure 3 describes the steps of BGA to reach the optimal solution set of PMUs to be installed. The main three operations of BGA, selection, crossover and mutation, are performed after setting up the appropriate initial conditions and elitism strategy presented in Figure 2. The binary version of each candidate PMU set go through the process until the best solution with the lowest NCE is achieved.

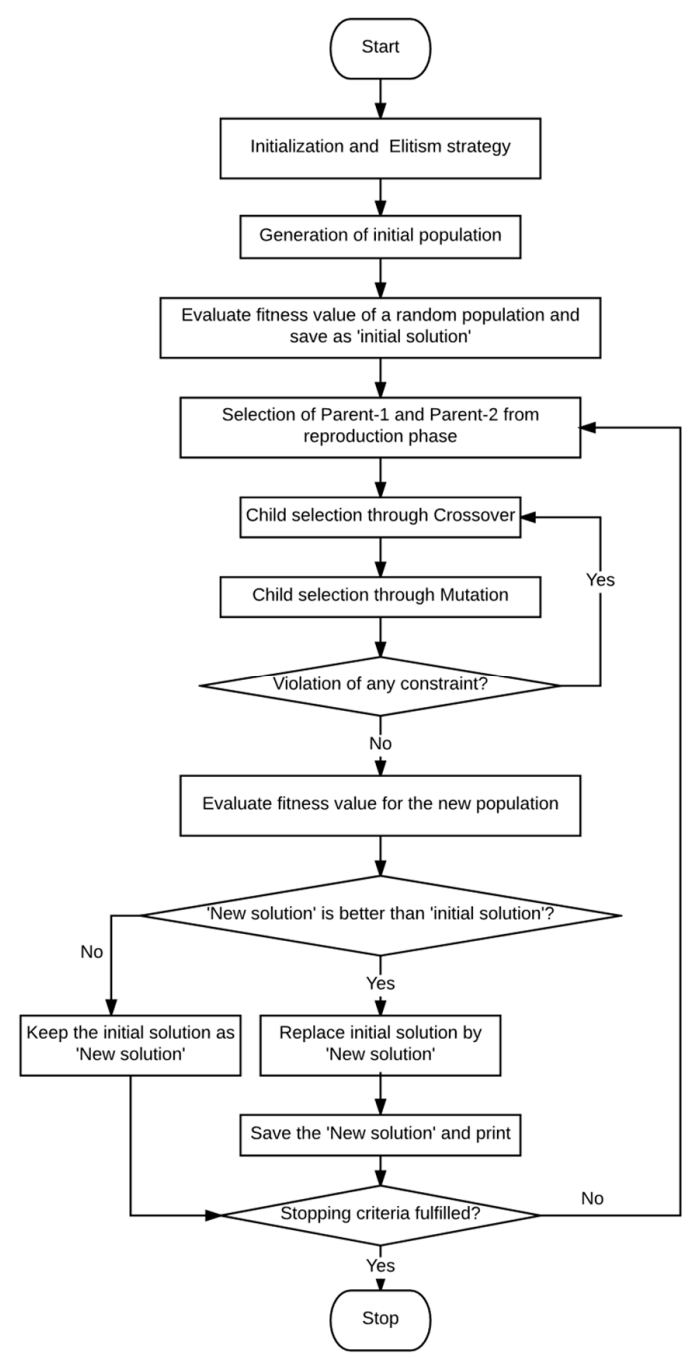

Figure 3. Flow diagram of binary genetic algorithm (BGA) steps. 


\section{Test Case Preparation}

To investigate the estimation performance for different PMU sets, two test cases with 30- and 118-buses are simulated. Before the inclusion of any PMU, the power system consists of SCADA meter measurements so that the network is observable. These measurements are mixed with white noise only and it is assumed that no bad-data is present in the system.

The details of the test cases with redundancy level, total number of measurements, and number of each types of SCADA meter are listed in Table 1 below.

Table 1. Details of 30- and 118-bus test cases.

\begin{tabular}{ccc}
\hline Measurement Description & 30-Bus System & 118-Bus System \\
\hline Total measurements & 126 & 440 \\
Redundancy & 2.13 & 1.87 \\
Number of voltage-magnitudes $(V m)$ & 14 & 62 \\
Number of real power-injections $(P G)$ & 16 & 55 \\
Number of reactive power-injections $(Q G)$ & 15 & 55 \\
Number of real power-flows from a bus $(P F)$ & 25 & 68 \\
Number of reactive power-flows from a bus $(Q F)$ & 24 & 68 \\
Number of real power-flows to a bus $(P T)$ & 16 & 66 \\
Number of reactive power-flows to a bus $(Q T)$ & 16 & 66 \\
\hline
\end{tabular}

The 30-bus test case has two critical measurements: PF_12-13 and QF_12-13. The 118-bus system has two critical measurements also: PF_39-40 and QF_39-40. So, bus-12 and bus-39 will be considered as elite genes at the time of optimization with the genetic algorithm for 30- and 118-bus systems, respectively, while covering the critical zones by PMUs.

SCADA measurement-based power systems described above are considered for finding out the optimal PMU locations. When a PMU is installed in a bus, it provides the corresponding bus-voltage magnitude and phase angle as well as the complex currents flowing from that bus $[4,5]$. This increases the redundancy level. Due to the low standard deviation (high weight) of a PMU meter reading, the estimator will always consider those readings in the estimation process. The additional PMU measurements for a specific set of PMUs are also shown in Table 2 below.

Table 2. Additional phasor measurement unit (PMU) meters for optimal PMU sets 1, 6, 24 for 30-bus system.

\begin{tabular}{cc}
\hline Measurement Type & Measurement Locations \\
\hline Voltage-magnitude buses & $1,6,24$ \\
Voltage-angle buses & $1,6,24$ \\
Current flow branches (real and reactive) & $1-2,1-3,6-7,6-8,6-9,6-10,6-28,24-25$ \\
\hline
\end{tabular}

Selection of standard deviation $(\sigma)$ is an important task in the design of WLS. The presumed values of $\sigma$ of various types of measurements are listed in Table 3. These values are kept fixed for all test cases.

The performance of the estimators is evaluated based on the NCE indicator, which is the accumulation of the absolute difference between all actual and estimated values of state variables as shown in Equations (5) and (6). The fitness function value of the BGA is the NCE value.

The performance of the genetic algorithm depends significantly upon the proper selection of population size, maximum iteration size, stopping criteria, probability of selection, mutation, and crossover. Higher values of iteration number and population size increase the probability of finding out the global solution. Besides, high crossover probability and low mutation probability will ensure the searching process avoids becoming trapped in local minima $[27,28]$. The number of crossover points could be higher for longer chromosomes to ensure quick saturation. A slack bus has been chosen as an elite chromosome in the GA optimization so that the reference angle of the 
measurements is accurate and remains the same. Details of the GA initialization are listed below in Table 4.

Table 3. Standard deviation for different measurement types [29].

\begin{tabular}{cc}
\hline Measurement Type & Standard Deviation $\sigma$ (Per Unit) \\
\hline SCADA voltage magnitude & 0.01 \\
Real power injection & 0.02 \\
Reactive power injection & 0.04 \\
Real power flow & 0.02 \\
Reactive power flow & 0.04 \\
PMU voltage magnitude & 0.0001 \\
PMU voltage angle & 0.006 \\
PMU current magnitude & 0.0001 \\
PMU current angle & 0.006 \\
\hline
\end{tabular}

Table 4. Details of genetic algorithm (GA) parameters.

\begin{tabular}{cc}
\hline Criteria & Considered Parameter \\
\hline Population size & 100 \\
\hline Selection probability & 1 \\
\hline Crossover probability & 0.9 \\
\hline Mutation probability & 0.25 \\
\hline Maximum number of iterations & 250 \\
\hline \multirow{2}{*}{ Elite chromosome (PMU must be installed) } & Slack bus: Bus-1 for 30-bus and bus-69 for 118-bus system \\
\cline { 2 - 2 } & Buses which cover the critical zones \\
\hline \multirow{2}{*}{ Crossover point } & 30-bus: Double \\
\cline { 2 - 2 } & 118-bus: Triple \\
\hline \multirow{2}{*}{ Stopping criteria } & 1. Reaching the maximum number of iterations \\
\cline { 2 - 2 } & 2. If the fitness value does not change for consecutive 100 iterations \\
\hline
\end{tabular}

\section{Results}

\subsection{Comparison with Heuristic Approach}

The two PMU-placement algorithms-heuristic and BGA-were tested on IEEE 30-bus and 118-bus systems. The results are arranged in Tables 5 and 6, respectively.

As can be observed from Table 5, placing two and five PMUs gave matched solutions for both algorithms. Beyond that, however, the BGA outperforms the heuristic. This happens because the heuristic technique places PMUs incrementally and cannot change the PMU locations selected in previous steps when searching for new buses to be equipped with PMUs. BGA, on the other hand, does not have such a restriction, and searches for the optimal solution as a set of PMUs and always converges to the global solution.

Table 5. OPP results for 30-bus power system.

\begin{tabular}{ccccc}
\hline \multirow{2}{*}{ Number of PMUs } & \multicolumn{2}{c}{ Heuristic } & BGA \\
\cline { 2 - 5 } & Optimal PMU Locations & $\begin{array}{c}\text { Normalized Cumulative } \\
\text { Error (NCE) Value }\end{array}$ & Optimal PMU Locations & NCE Value \\
\hline 2 & 1,6 & $2.89 \times 10^{-4}$ & 1,6 & $2.89 \times 10^{-4}$ \\
3 & $1,6,25$ & $1.81 \times 10^{-4}$ & $1,10,25$ & $1.78 \times 10^{-4}$ \\
4 & $1,6,22,25$ & $1.28 \times 10^{-4}$ & $1,6,12,24$ & $1.27 \times 10^{-4}$ \\
5 & $1,6,12,22,25$ & $7.69 \times 10^{-5}$ & $1,6,12,22,25$ & $7.69 \times 10^{-5}$ \\
\hline
\end{tabular}


Table 6. OPP results for 118-bus power system.

\begin{tabular}{ccccc}
\hline \multirow{2}{*}{ Number of PMUs Heuristic } & \multicolumn{2}{c}{ BGA } \\
\cline { 2 - 5 } & Optimal PMU Locations & NCE Value & Optimal PMU Locations & NCE Value \\
\hline 2 & 69,100 & $6.35 \times 10^{-4}$ & 69,100 & $6.35 \times 10^{-4}$ \\
3 & $69,100,30$ & $5.41 \times 10^{-4}$ & $69,38,26$ & $5.397 \times 10^{-4}$ \\
4 & $69,100,30,25$ & $4.63 \times 10^{-4}$ & $26,38,69,100$ & $4.362 \times 10^{-4}$ \\
5 & $69,100,30,25,64$ & $3.83 \times 10^{-4}$ & $69,100,30,25,64$ & $3.83 \times 10^{-4}$ \\
6 & $69,100,30,25,64,32$ & $3.32 \times 10^{-4}$ & $69,100,30,25,64,32$ & $3.32 \times 10^{-4}$ \\
7 & $69,100,30,25,64,32,1$ & $2.97 \times 10^{-4}$ & $69,100,30,25,64,32,1$ & $2.97 \times 10^{-4}$ \\
\hline
\end{tabular}

Figure 4 shows how the proposed approach with GA reaches a global solution in searching for the optimal PMU set. Optimization for 5 PMUs in the 30-bus system is shown in the figure and it is seen that the NCE indicator keeps decreasing until it finds the best set of PMUs with the lowest indicator $\left(7.69 \times 10^{-5}\right)$. Iteration stops before the maximum number of iterations (250) because the stopping criteria resulting in the same NCE indicator for 100 iterations was fulfilled.

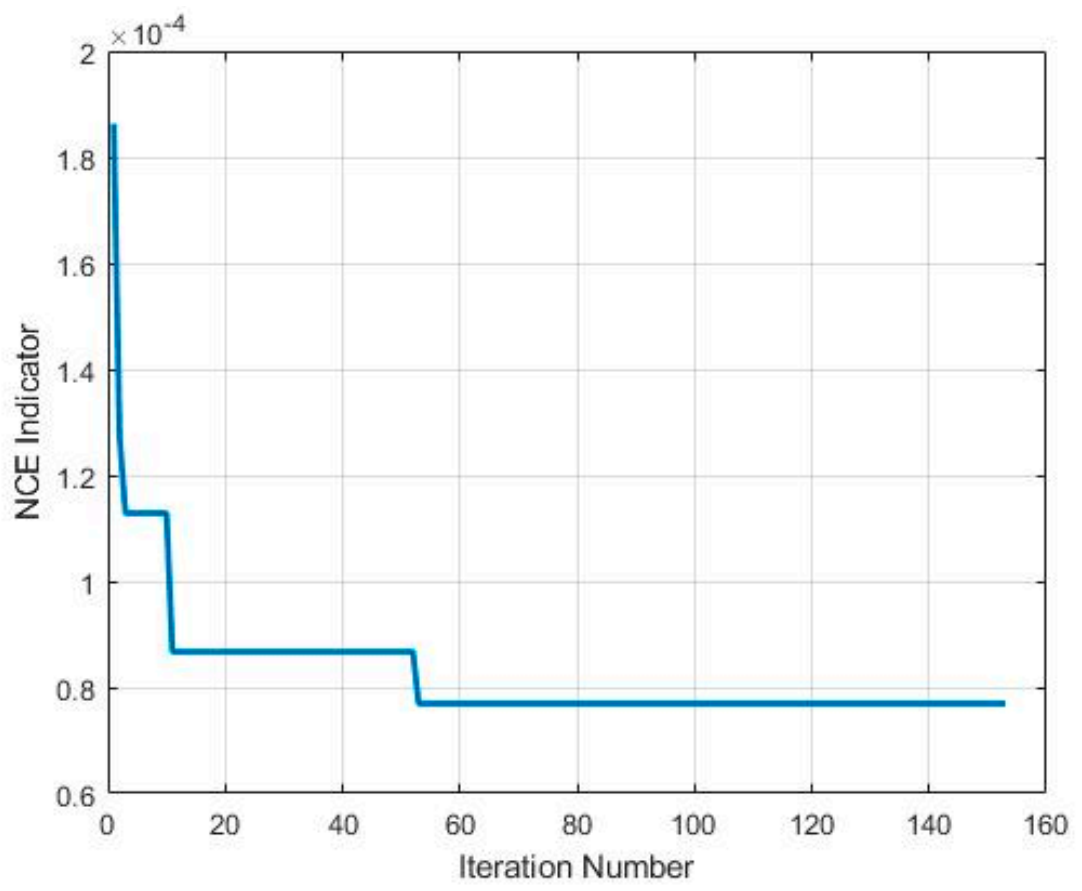

Figure 4. Convergence plot for IEEE 30-bus system with 5-PMU placement.

Results of 118-bus system in Table 6 confirms the superiority of the BGA over the heuristic in locating global optimal PMU locations for any system regardless of its size. The results of optimizing 3 and 4 PMUs confirm that the heuristic approach often falls in local optima.

\subsection{Optimal PMU Locations by Covering Critical Zones}

The paper proposes to check the presence of critical measurements in the test case and thus place PMUs in the critical zones. The optimal PMU locations for a 30-bus system are listed below in Table 7 where both cases for covering and non-covering critical zones are presented for a different number of PMU placements. Figure 5 presents the results of Table 7 for better illustration of the simulation results. Optimal solution sets by BGA with 2 to 5 PMUs are presented with corresponding NCE indicator values. 
Table 7. OPP results for 30-bus power system.

\begin{tabular}{|c|c|c|c|c|}
\hline Number of PMUs & \multicolumn{2}{|c|}{ NCC (Not Covering Critical) } & \multicolumn{2}{|c|}{ CC (Covering Critical) } \\
\hline 2 & 1,6 & $2.89 \times 10^{-4}$ & 1,12 & $4.5202 \times 10^{-4}$ \\
\hline 4 & $1,6,12,24$ & $1.2792 \times 10^{-4}$ & $1,6,12,24$ & $1.2792 \times 10^{-4}$ \\
\hline 5 & $1,6,25,22,12$ & $7.69 \times 10^{-5}$ & $1,6,25,22,12$ & $7.69 \times 10^{-5}$ \\
\hline
\end{tabular}

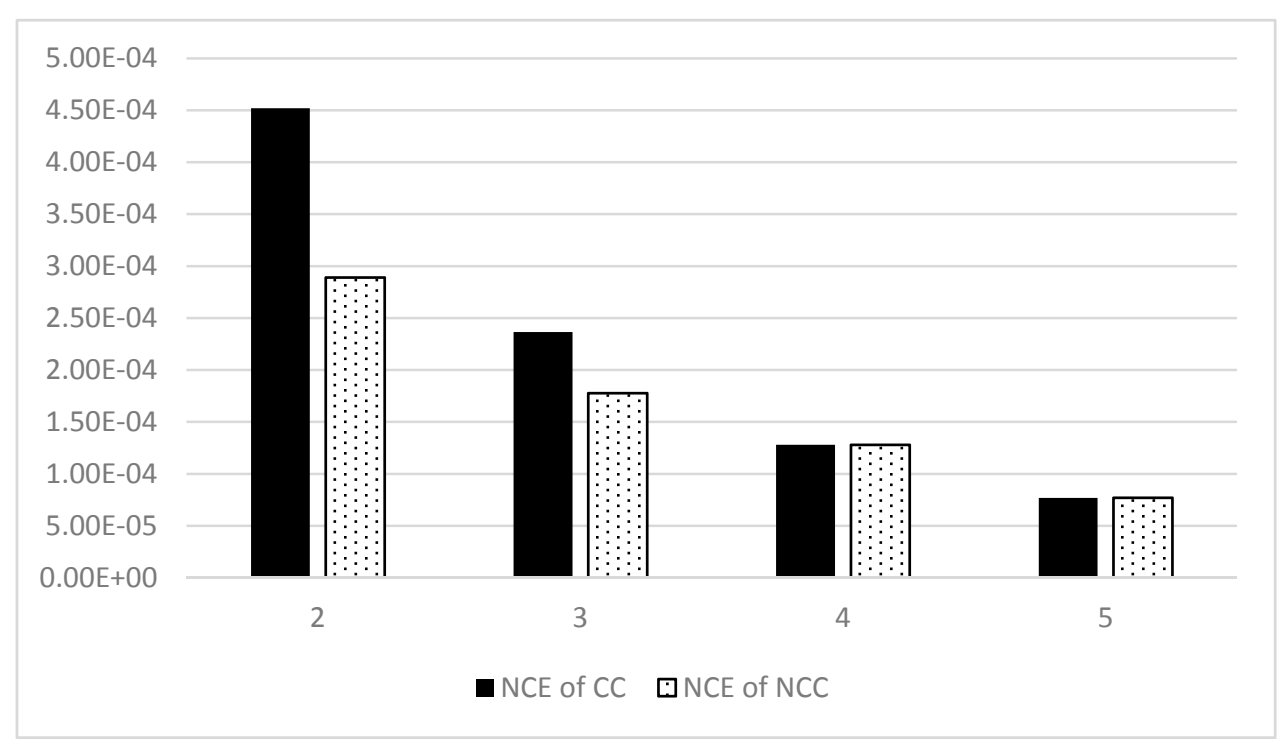

Figure 5. Comparison of normalized cumulative error (NCE) values of covering critical (CC) and not covering critical (NCC) for different numbers of PMUs.

As seen from the above table and the graph, the indicator value keeps decreasing with the increase of PMU number. For the sets with 2 and 3 PMUs, the indicator value for CC is higher than that of NCC. This occurs because of the constraint of the optimization technique in covering the critical bus which is not included in the global solution sets given by NCC. For example, while placing 3 PMUs, solution set $\{1,10,25\}$ is the global solution with lowest indicator $1.7755 \times 10^{-4}$ which is not achieved by the CC solution set $\{1,6,12\}$ because it forces one PMU to be installed at the critical bus-12. Optimization for placing 4 and 5 PMUs results in the same solution for both NCC and CC cases. It reflects the global solutions for both.

Optimal solution sets of PMUs for the 118-bus system are listed in Table 8 above. The 2-PMU set to 7-PMU set solutions are shown for both NCC and CC conditions. All the CC solutions are different from those of NCC. Even though the indicators for the CC cases are higher, it is recommended this solution set is chosen to install the PMUs for higher robustness.

Table 8. OPP results for 118-bus power system.

\begin{tabular}{ccccc}
\hline \multirow{2}{*}{ Number of PMUs } & \multicolumn{2}{c}{ NCC (Not Covering Critical) } & \multicolumn{2}{c}{ CC (Covering Critical) } \\
\cline { 2 - 5 } & Optimal PMU Locations & NCE Value & Optimal PMU Locations & NCE Value \\
\hline 2 & 69,100 & $6.35 \times 10^{-4}$ & 39,69 & $7.095 \times 10^{-4}$ \\
3 & $69,38,26$ & $5.396 \times 10^{-4}$ & $39,69,100$ & $6.08 \times 10^{-4}$ \\
4 & $26,38,69,100$ & $4.362 \times 10^{-4}$ & $39,49,69,100$ & $5.226 \times 10^{-4}$ \\
5 & $69,100,30,25,64$ & $3.83 \times 10^{-4}$ & $39,69,31,100,116$ & $4.699 \times 10^{-4}$ \\
6 & $69,100,30,25,64,32$ & $3.32 \times 10^{-4}$ & $39,69,26,29,38,100$ & $4.12 \times 10^{-4}$ \\
7 & $69,100,30,25,64,32,1$ & $2.97 \times 10^{-4}$ & $39,69,12,61,19,100,32$ & $3.59 \times 10^{-4}$ \\
\hline
\end{tabular}




\subsection{Optimization when the Critical Zones are Non-Interacting}

The test cases used for this paper (Table 1) has the critical measurements in the same locations. For example, the 30-bus system test case has two critical measurements: PF_12-13 and QF_12-13. Now, a modified test case is prepared for 30-bus system with much lower redundancy (1.74) which has the critical measurements in different zones of the system. The optimization results for such a case is presented below in Table 9.

Table 9. OPP results for 30-bus system with non-interacting critical zones.

\begin{tabular}{ccccc}
\hline Critical Measurements & Elite Chromosomes & \multicolumn{3}{c}{ Optimization Results } \\
\hline & & No of PMUs & Optimal PMU Locations & NCE Indicator \\
\cline { 2 - 5 } PF_12-13, & 2 & 1,12 & $8.198 \times 10^{-4}$ \\
QF_12-13, & 1 (slack bus), 12, 26 & 3 & $1,12,26$ & $6.707 \times 10^{-4}$ \\
QF_25-26, & & 4 & $1,6,12,26$ & $4.004 \times 10^{-4}$ \\
Vm_26 & 5 & $1,6,12,24,26$ & $2.245 \times 10^{-4}$ \\
& & 6 & $1,6,12,24,26,4$ & $1.011 \times 10^{-4}$ \\
\hline
\end{tabular}

Two PMUs are needed now to cover the critical zones: 12 and 26. While placing 2 PMUs, the solution will be the set with lowest NCE value among the possible combinations. It is noticeable that the NCE values of this presented case are higher than the values of the CC case in Table 7. This is because of the lower redundancy level.

\subsection{Significance of Covering Critical Locations}

The reason for imposing the constraint of placing PMUs in critical locations is to reduce the impact of bad data on the estimation performance. Bad data could come from any location of the system. If it occurs in a critical location or around it, the impact will be severe, and the estimation performance will be significantly disturbed. Covering the critical location in PMU optimization will ensure that the bad-data presence, even around the critical location, will not hamper the estimation performance of the system. Table 10 presents the impact of bad-data presence on estimation performance for both CC and NCC scenarios. Figure 6 depicts the results of Table 10 clearly in a bar chart.

Table 10. Bad-data investigation with 3-PMU case of 30-bus.

\begin{tabular}{cccc}
\hline Cases & Bad-Data Location & $\begin{array}{c}\text { NCC (Not Covering Critical) } \\
\text { (PMUs in 1, 10, 25) }\end{array}$ & $\begin{array}{c}\text { CC (Covering Critical) } \\
\text { (PMUs in 1, 6,12) }\end{array}$ \\
\hline White noises only & N/A & $1.7755 \times 10^{-4}$ & $2.3653 \times 10^{-4}$ \\
\hline & Vm_12 & $1.155 \times 10^{-3}$ & $2.370 \times 10^{-4}$ \\
Single bad-data around & QT_15-12 & $6.733 \times 10^{-4}$ & $2.415 \times 10^{-4}$ \\
critical locations & PF_12-14 & $6.294 \times 10^{-4}$ & $2.358 \times 10^{-4}$ \\
& PF_12-16 & $1.035 \times 10^{-3}$ & $2.366 \times 10^{-4}$ \\
& QF_12-14 & $2.453 \times 10^{-4}$ & $2.373 \times 10^{-4}$ \\
& QF_12-16 & $3.157 \times 10^{-4}$ & $2.375 \times 10^{-4}$ \\
\hline
\end{tabular}

The 30-bus test system had two critical measurements: PF_12-13 and QF_12-13. That is why a PMU is placed in bus 12 to cover the critical zone. It can be seen from the table above that the estimation performance for the NCC optimal set $\{1,10,25\}$ is better than the CC optimal set $\{1,6,12\}$ with white noise only. But the presence of a single bad data around bus 12 has completely changed the scenario. Because of the effect of bad data, the estimation performance for NCC deteriorates significantly even after having 3 PMUs installed. The error indicator is raised because of bad data in the NCC case. However, the other case of optimization shows robust behavior with bad data due to the presence of a PMU at bus-12 where the NCE remains constant at around $2.3653 \times 10^{-4}$ for all the cases. 


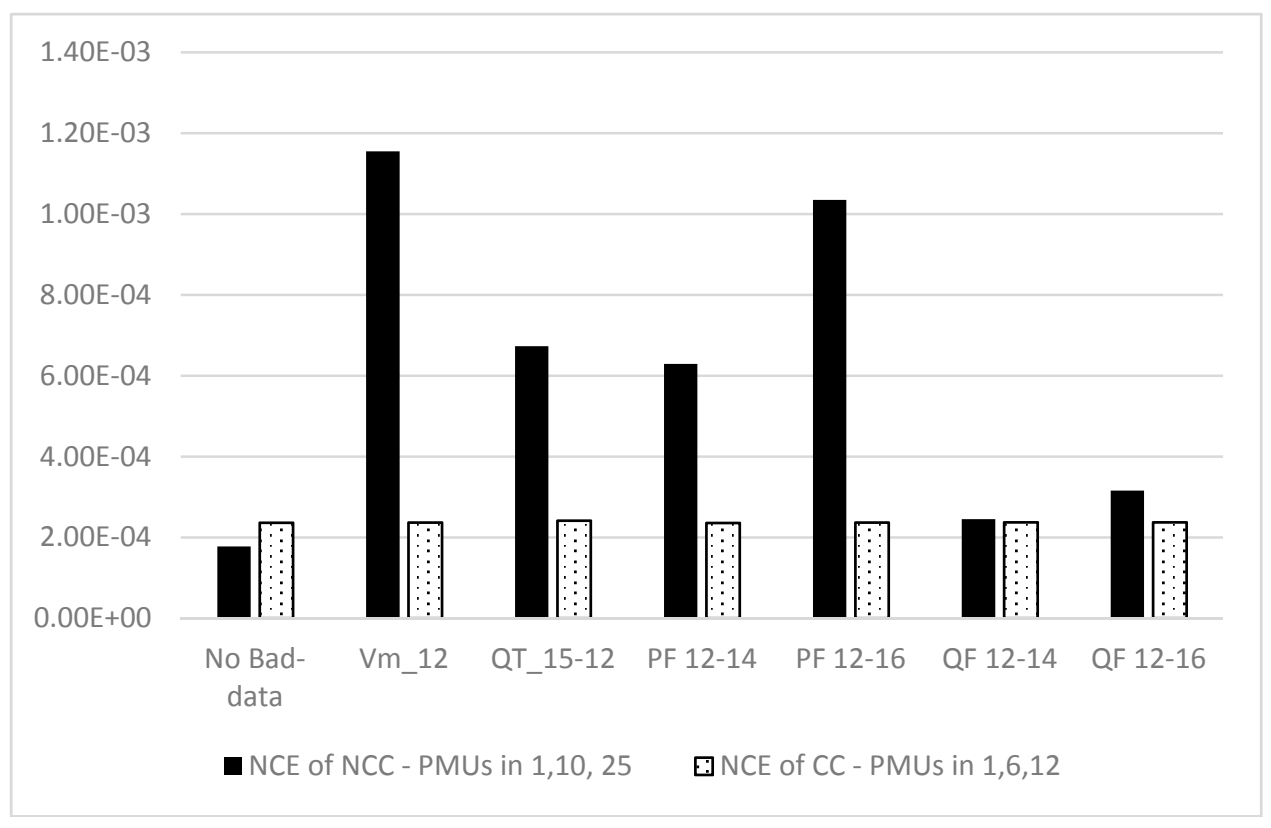

Figure 6. The impact of PMU placement in the critical zone under the bad-data constraint.

\section{Conclusions}

This paper presents a novel approach of PMU placement in power systems. The binary genetic algorithm is used to solve the proposed optimization problem which aims to find the optimal set of PMUs to be installed in a SCADA measurement-based power system. It is seen that the BGA performs better than the heuristic approach in reaching the global solution. The objective of the optimization is to guarantee the best performance of state estimation. It also ensures critical zones are covered by PMUs and thus the least impact of the presence of bad data in the estimation performance. Optimal solution sets for both covering and non-covering critical zones are presented. Some of the optimal solution sets are found to be identical in both approaches, indicating that both techniques reached the global solution. Covering the critical locations sometimes results in the non-global solution, but the solution is robust and the least sensitive to the presence of bad data. Results of the optimal solution sets and the corresponding NCE indicators for 30- and 118-bus power systems verify the effectiveness of the proposed method irrespective of the power-system size. In practical scenarios where the modern-day power systems are inclining towards installing PMU meters with an existing system, such a strategy of getting the most optimal locations for any limited number of PMUs would be very useful and effective for planning engineers.

Acknowledgments: The authors acknowledge the Information Technology Center at King Fahd University of Petroleum and Minerals for providing high-performance computing resources that have contributed to the research results reported within this paper.

Author Contributions: Mohammad Shoaib Shahriar and Ibrahim Omar Habiballah conceived and designed the research idea; Mohammad Shoaib Shahriar and Huthaifa Hussein worked with the simulation and wrote the paper in collaboration. Ibrahim Omar Habiballah proofread the paper.

Conflicts of Interest: The authors declare no conflict of interest. 


\section{References}

1. Hurtgen, M.; Maun, J.-C. Advantages of power system state estimation using Phasor Measurement Units. In Proceedings of the 16th Power Systems Computation Conference (PSCC), Glasgow, Scotland, UK, 14-18 July 2008; Curran Associates: Red Hook, NY, USA, 2010; pp. 1161-1167.

2. Gómez-Expósito, A.; Abur, A.; Rousseaux, P.; Jaén, A.; Gómez-Quiles, C. On the Use of PMUs in Power System State Estimation. In Proceedings of the 17th Power Systems Computation Conference (PSCC), Stockholm, Sweden, 22-26 August 2011; Curran Associates: Red Hook, NY, USA, 2012; pp. 1080-1092.

3. Manousakis, N.M.; Korres, G.N.; Georgilakis, P.S. Optimal placement of phasor measurement units: A literature review. In Proceedings of the 16th International Conference on Intelligent System Applications to Power Systems (ISAP), Hersonissos, Greece, 25-28 September 2011; IEEE: Piscataway, NJ, USA, 2011; pp. 1-6.

4. More, K.K.; Jadhav, H.T. A literature review on optimal placement of phasor measurement units. In Proceedings of the International Conference on Power, Energy and Control (ICPEC), Dindigul, India, 6-8 February 2013; IEEE: Piscataway, NJ, USA, 2013; pp. 220-224.

5. Nazari-Heris, M.; Mohammadi-Ivatloo, B. Application of heuristic algorithms to optimal PMU placement in electric power systems: An updated review. Renew. Sustain. Energy Rev. 2015, 50, 214-228. [CrossRef]

6. Negash, K.; Khan, B.; Yohannes, E. Artificial Intelligence Versus Conventional Mathematical Techniques: A Review for Optimal Placement of Phasor Measurement Units. Technol. Econ. Smart Grids Sustain. Energy 2016, 1, 10. [CrossRef]

7. Aminifar, F.; Fotuhi-Firuzabad, M.; Safdarian, A.; Davoudi, A.; Shahidehpour, M. Synchrophasor Measurement Technology in Power Systems: Panorama and State-of-the-Art. IEEE Access 2014, 2, 1607-1628. [CrossRef]

8. Chakhchoukh, Y.; Vittal, V.; Heydt, G.T.; Ishii, H. LTS-Based Robust Hybrid SE Integrating Correlation. IEEE Trans. Power Syst. 2017, 32, 3127-3135. [CrossRef]

9. Zivanovic, R.; Cairns, C. Implementation of PMU technology in state estimation: An overview. In Proceedings of the IEEE AFRICON 4th Conference in Africa, Stellenbosch, South Africa, 27 September 1996; IEEE: Piscataway, NJ, USA, 2002; pp. 1006-1011.

10. Zhou, M.; Centeno, V.A.; Thorp, J.S.; Phadke, A.G. An Alternative for Including Phasor Measurements in State Estimators. IEEE Trans. Power Syst. 2006, 21, 1930-1937. [CrossRef]

11. Nuqui, R.F.; Phadke, A.G. Hybrid Linear State Estimation Utilizing Synchronized Phasor Measurements. In Proceedings of the IEEE Lausanne PowerTech, Lausanne, Switzerland, 1-5 July 2007; IEEE: Piscataway, NJ, USA, 2008; pp. 1665-1669.

12. Abbasy, N.H.; Ismail, H.M. A Unified Approach for the Optimal PMU Location for Power System State Estimation. IEEE Trans. Power Syst. 2009, 24, 806-813. [CrossRef]

13. Zhu, K.; Nordstrom, L.; Ekstam, L. Application and analysis of optimum PMU placement methods with application to state estimation accuracy. In Proceedings of the IEEE Power \& Energy Society General Meeting, Calgary, AB, Canada, 26-30 July 2009; IEEE: Piscataway, NJ, USA, 2009; pp. 1-7.

14. Celik, M.K.; Liu, W.-H.E. An incremental measurement placement algorithm for state estimation. IEEE Trans. Power Syst. 1995, 10, 1698-1703. [CrossRef]

15. Li, Q.; Negi, R.; Ilić, M.D. Phasor measurement units placement for power system state estimation: A greedy approach. In Proceedings of the IEEE Power and Energy Society General Meeting, Detroit, MI, USA, 24-29 July 2011; IEEE: Piscataway, NJ, USA, 2011; pp. 1-8.

16. Yucra, M.; Schmidt, F.; de Almeida, M.C. A comparative analysis of inclusion of PMUs in the power system state estimator. In Proceedings of the IEEE 6th Latin American Symposium on Circuits \& Systems (LASCAS), Montevideo, Uruguay, 24-27 February 2015; IEEE: Piscataway, NJ, USA, 2015; pp. 1-4.

17. Göl, M.; Abur, A. PMU placement for robust state estimation. In Proceedings of the North American Power Symposium (NAPS), Manhattan, KS, USA, 22-24 September 2013; IEEE: Piscataway, NJ, USA, 2013; pp. 1-5.

18. Wu, J.; Xiong, J.; Shil, P.; Shi, Y. Optimal PMU placement for identification of multiple power line outages in smart grids. In Proceedings of the IEEE 57th International Midwest Symposium on Circuits and Systems (MWSCAS), College Station, TX, USA, 3-6 August 2014; IEEE: Piscataway, NJ, USA, 2014; pp. 354-357.

19. Lu, Z.-G.; Tian, S.-S.; Liu, X.-Y.; Zhao, H. A new numerical algorithm to check the observability and bad-data processing ability of the metering scheme. Int. J. Electr. Power Energy Syst. 2015, 66, 144-153. [CrossRef] 
20. Gou, B.; Kavasseri, R.G. Unified PMU Placement for Observability and Bad Data Detection in State Estimation. IEEE Trans Power Syst. 2014, 29, 2573-2580. [CrossRef]

21. Chen, J.; Liao, Y. Optimal Placement of Phasor Measurement Units for Improving Power System State Estimation Accuracy: A Heuristic Approach. J. Electr. Eng. Electron. Technol. 2012, 1. [CrossRef]

22. Shahriar, M.S.; Ahmad, F.A.; Habiballah, I.O.; Asif, M.A.; Mukherjee, S. Artificial Bee Colony based Optimal PMU Placement in Power System State Estimation. In Proceedings of the 1st International Conference on Advanced Information and Communication Technology (ICAICT), Chittagong, Bangladesh, 16-17 May 2016.

23. Abur, A.; Gómez-Expósito, A. Power System State Estimation: Theory and Implementation, 1st ed.; Marcel Dekker: New York, NY, USA, 2004; ISBN 0-8247-5570-7.

24. Zimmerman, R.D.; Murillo-Sanchez, C.E.; Thomas, R.J. MATPOWER: Steady-State Operations, Planning and Analysis Tools for Power Systems Research and Education. IEEE Trans. Power Syst. 2011, 26, 12-19. [CrossRef]

25. Göl, M.; Abur, A. LAV Based Robust State Estimation for Systems Measured by PMUs. IEEE Trans. Smart Grid 2014, 5, 1808-1814. [CrossRef]

26. Do Coutto Filho, M.B.; de Souza, J.C.S.; de Oliveira, F.M.F.; Schilling, M.T. Identifying critical measurements and sets for power system state estimation. In Proceedings of the IEEE Porto Power Tech Proceedings, Porto, Portugal, 10-13 September 2001; IEEE: Piscataway, NJ, USA, 2002; pp. 6-12.

27. Houck, C.R.; Joines, J.A.; Kay, M.G. A Genetic Algorithm for Function Optimization: A Matlab Implementation; Technical Report NCSU-IE TR-95-09; North Carolina State University: Raleigh, NC, USA, 1995.

28. Schmitt, L.M. Theory of genetic algorithms. Theor. Comput. Sci. 2001, 259, 1-61. [CrossRef]

29. Kamireddy, S. Comparison of State Estimation Algorithms Considering Phasor Measurement Units and Major and Minor Data Loss. Master's Thesis, Mississippi State University, Starkville, MS, USA, 2008.

(C) 2018 by the authors. Licensee MDPI, Basel, Switzerland. This article is an open access article distributed under the terms and conditions of the Creative Commons Attribution (CC BY) license (http:/ / creativecommons.org/licenses/by/4.0/). 